\title{
ESTUDO COMPARATIVO DE INDICADORES NUTRICIONAIS EM PACIENTES COM NEOPLASIAS DO TRATO DIGESTÓRIO
}

\section{Comparative study of nutritional status indicators among patients with gastrointes- tinal neoplasms}

\author{
Vânia Aparecida LEANDRO-MERHI ${ }^{1}$, Ana Paula TRISTÃO ${ }^{1}$, Maria Clara MORETTO ${ }^{1}$, \\ Natália Maria FUGULIN ${ }^{1}$, Kátia Cristina PORTERO-McLELLAN ${ }^{1}$, José Luiz Braga de AQUINO²
}

ABCDDV/606

Leandro-Merhi VA, Tristão AP, Moretto MC, Fugulin NM, Portero-McLellan KC, Aquino JLB. Estudo comparativo de indicadores nutricionais em pacientes com neoplasias do trato digestório. ABCD Arq Bras Cir Dig 2008;21(3):114-9

RESUMO - Racional - O câncer tem sido considerado atualmente como um dos principais problemas de saúde pública em todo o mundo e os tumores mais frequentes são os dos tratos gastrintestinal e o respiratório. Objetivo - Comparar indicadores do estado nutricional em pacientes portadores de neoplasias do trato digestório. Método - Estudo comparativo de indicadores nutricionais (antropométricos, laboratoriais e dietéticos), entre 28 pacientes portadores de neoplasias do trato digestório (esofágicas, gástricas e intestinais), em um hospital universitário. Resultados - Não foi encontrada diferença significativa entre os três grupos de neoplasia para os indicadores laboratoriais, de consumo alimentar e antropométricos, exceto para a circunferência do braço $(P=0,0471)$. A perda de peso ocorrida durante a internação foi observada em $46,4 \%$ dos pacientes estudados e a dieta enteral foi a prescrição dietética mais prevalente entre os pacientes, especialmente nos portadores de neoplasia de esôfago. Conclusão Pacientes com diagnóstico de neoplasia de trato digestório apresentam comprometimento no estado nutricional e necessitam de acompanhamento nutricional no decorrer do tratamento destas enfermidades.

DESCRITORES - Neoplasias esofágicas. Neoplasias abdominais. Neoplasias do colo. Estado nutricional.

\section{INTRODUÇÃO}

O câncer tem sido considerado atualmente como um dos principais problemas de saúde pública em todo o mundo ${ }^{17}$. A alta mortalidade vem crescendo nos últimos anos em decorrência do envelhecimento populacional, do processo de industrialização e das modificações do estilo de vida. Alguns estudos têm mostrado que o diagnóstico de neoplasias surge geralmente após os 50 anos de idade, sendo que os tumores mais frequentes são os dos tratos gastrintestinal e respiratório, e os órgãos mais afetados são o intestino delgado, apêndice cecal e reto ${ }^{10,15,32}$.

No Brasil, segundo o INCA ${ }^{19}$, a estimativa de casos novos de câncer de esôfago em 2008, foi de 7.900 em homens e de 2.650 em mulheres ${ }^{19}$. No câncer de estômago, a estimativa foi de 14.080 entre homens e de 7.720 nas mulheres, configurando-se no mundo como a quarta causa mais comum e, como segunda causa de óbitos por câncer. A estimativa de novos casos de câncer de cólon e reto é de 12.490 em homens e de 14.500 em mulheres. Quanto à incidência, o câncer de cólon e reto é a terceira causa mais comum de câncer no mundo em ambos os sexos é a segunda causa nos países desenvolvidos, com padrões geográficos similares em ambos os sexos. No entanto, é cerca de 20 a

Trabalho realizado nas Faculdade de ${ }^{1}$ Nutrição e ${ }^{2}$ Medicina da UNICAMP, Campinas, SP, Brasil

Correspondência: Vânia Ap. Leandro-Merhi, e-mail: valm@ dglnet.com.br
$50 \%$ maior em homens na maioria das populações ${ }^{19}$.

No que se refere ao estado nutricional destes pacientes, a perda de peso relacionada ao câncer é bem conhecida pelo fato de contribuir para a piora do bem estar do paciente, redução da tolerância às drogas antineoplásicas e piora do prognóstico $^{9,21}$. É observada a síndrome wasting ${ }^{21}$, a qual pode ser caracterizada por situações de perda ponderal e de massa magra, que vem acompanhada de alterações na ingestão energético-protéica, disfagia, odinofagia, xerostomia, disgeusia, obstipação intestinal, náuseas, vômitos, diarréia, sensação de empachamento ou plenitude pós-prandial, comprometimento funcional e também alterações metabólicas sistêmicas 9 .

Frente às alterações nutricionais que comprometem esta população e que podem repercutir no curso clínico da doença, este trabalho teve como objetivo comparar os indicadores do estado nutricional (antropométricos, laboratoriais e dietéticos), entre pacientes portadores de neoplasias esofágicas, gástricas e intestinais.

\section{MÉTODO}

Os pacientes incluídos neste estudo foram selecionados por meio de um banco de dados pertencente ao projeto "Estado nutricional de indivíduos portadores de neoplasias do trato digestório", tendo obtido parecer favorável do Comitê de Ética em Pesquisa da Pontifícia Universidade Católica de Campinas, SP, Brasil (Protocolo no 201/07). O estudo foi do tipo transversal, com 28 pacientes adultos de ambos os 
sexos, portadores de neoplasias do trato-digestório - esôfago $(\mathrm{n}=8)$, estômago $(\mathrm{n}=8)$ e intestinais: cólon e reto $(\mathrm{n}=12)$-, internados na Clínica Cirúrgica do Hospital e Maternidade Celso Pierro, da PUC-Campinas, Campinas,SP, Brasil durante o ano de 2007. Os dados foram coletados por meio de um protocolo de atendimento nutricional previamente definido, sendo preenchidas as informações de identificação e diagnóstico a partir do prontuário médico. Este era composto por dados de identificação e antecedentes pessoais, indicadores antropométricos, laboratoriais e dietéticos (dieta habitual do paciente, que consiste na descrição da alimentação representativa de um dia alimentar), prescrição da dieta, necessidades energéticas e a conduta dietoterápica adotada.

Os indicadores antropométricos avaliados foram o peso atual (PA), altura (A), circunferência braquial (CB) e prega cutânea triciptal (PCT). A partir destas medidas foram calculados o índice de massa corporal (IMC), a circunferência muscular do braço (CMB), a área muscular do braço (AMB) e a área adiposa do braço (AAB). A classificação das medidas de PCT, CMB, AMB e AAB foram realizadas de acordo os critérios estabelecidos na literatura ${ }^{12,13,16,18}$. Para os pacientes com mais de 74,9 anos foram utilizados os parâmetros antropométricos estabelecidos por Burr \& Phillips, $1984^{2}$. O índice de massa corporal (IMC), calculado por meio do quociente peso/estatura ${ }^{2}$ - peso corporal expresso em quilogramas $(\mathrm{kg})$ e estatura em metros (m) -, foi classificado obedecendo aos critérios estabelecidos pela WHO, $1998^{31}$ para adultos (até 60 anos) e Lipschitz, $1994^{22}$ para idosos (> 60 anos).

Para a análise dos indicadores laboratoriais de linfócitos, leucócitos, eritrócitos, hemoglobina e hematócrito, utilizaram-se como ponto de corte os valores esperados preconizados por Fleury, $2008^{11}$.

Para avaliar a ingestão alimentar utilizou-se a análise de um dia alimentar habitual. A composição centesimal dos alimentos presentes na anamnese alimentar foi calculada no software NutWin ${ }^{\circledR}$. Assim, foi calculado o consumo energético habitual (CEH), adotando-se os seguintes critérios de classificação: até $1000 \mathrm{kcal} ;>1000$ a $1500 \mathrm{kcal}$; $>1500$ a $2000 \mathrm{kcal} \mathrm{e}>2000 \mathrm{kcal}$. A estimativa do gasto energético total (GET) foi obtida por meio da Equação de Harris Bennedict, sendo também calculada a porcentagem de adequação do CEH em relação ao GET. A classificação da dieta prescrita para cada paciente seguiu a padronização do Serviço de Nutrição e Dietética do hospital, sendo: dieta geral, branda, leve, líquida, enteral e jejum. Os tipos de dieta prescritos aos pacientes foram levantados de acordo com as prescrições médicas, descritos nos prontuários.

Os dados relativos à alteração ponderal (manutenção, ganho ou perda ponderal) e à intervenção nutricional (no momento da internação, durante a internação e na alta hospitalar) foram coletados a partir dos protocolos de atendimento nutricional, aplicados rotineiramente nos atendimentos aos pacientes. Por fim, todos estes indicadores foram comparados entre os pacientes portadores de neoplasias esofágicas, gástricas e intestinais.

Os dados foram processados pelo programa Excel e expressos por meio de estatística descritiva. A seguir foram analisados pelo programa computacional SAS (Statistical
Analysis System ${ }^{28}$. A avaliação dos dados foi realizada por meio de análise descritiva. Para a comparação de proporções foi aplicado o teste exato de Fisher, e para a comparação de medidas contínuas ou ordenáveis entre três grupos foi aplicado o teste de Kruskal-Wallis. O nível de significância adotado para os testes estatísticos foi de $5 \%(P<0,005)$.

\section{RESULTADOS}

A maioria dos pacientes portadores de neoplasias era do sexo masculino $(60,8 \%)$ e a idade média foi de $59,9 \pm 14,6$ anos. No sexo feminino $(39,2 \%)$ prevaleceu a neoplasia coloretal $(66,6 \%)$ e no masculino a esofágica foi a mais frequente $(87,5 \%)$. A média do tempo de internação entre todos os pacientes foi de $11,3 \pm 6,3$ dias, sendo que, os portadores de neoplasias esofágicas apresentaram maior tempo de internação (15,1 $\pm 5,8$ dias), no entanto, sem diferença significativa $(P=0,0531)$. Os pacientes com neoplasias gástricas foram os que apresentaram menor tempo de internação, com $8,8 \pm 8$ dias. Os valores médios dos indicadores antropométricos foram semelhantes entre os pacientes, independentemente do tipo de neoplasia, exceto a $\mathrm{CB}$ que foi maior nos pacientes com neoplasia de cólon e reto $(P=0,0471)$ (Tabela 1$)$.

TABELA 1 - Indicadores antropométricos e características da população estudada, de acordo com o tipo de neoplasia.

\begin{tabular}{|c|c|c|c|c|c|}
\hline & & & eoplasias & & \\
\hline & $\begin{array}{c}\text { Esôfago } \\
(\mathrm{n}=8)\end{array}$ & $\begin{array}{l}\text { Estômago } \\
\quad(n=8)\end{array}$ & $\begin{array}{c}\text { Cólon e Reto } \\
(n=12)\end{array}$ & $\begin{array}{c}\text { Total } \\
(n=28)\end{array}$ & $P$ \\
\hline Sexo & & & & & \\
\hline Feminino (\%) & $1(12,5)$ & $2(25,0)$ & $8(66,6)$ & $11(39,2)$ & $0,0540^{*}$ \\
\hline Masculino (\%) & $7(87,5)$ & $6(75,0)$ & $4(33,3)$ & $17(60,8)$ & \\
\hline Idade (anos) & & & & & \\
\hline$\chi \pm \mathrm{DP}$ & $57,1 \pm 10,3$ & $59,6 \pm 15,2$ & $59,3 \pm 14,64$ & $59,9 \pm 14,6$ & $0,9491^{* *}$ \\
\hline Mediana & 56,5 & 60,0 & 57,0 & 57,0 & \\
\hline TI (dias) & & & & & \\
\hline$\chi \pm \mathrm{DP}$ & $15,1 \pm 5,8$ & $8,8 \pm 8,0$ & $12,3 \pm 5,4$ & $11,3 \pm 6,3$ & $0,0531^{* *}$ \\
\hline Mediana & 15,5 & 6,0 & 11,0 & 10,5 & \\
\hline IMC $\left(\mathrm{Kg} / \mathbf{m}^{2}\right)$ & & & & & \\
\hline$\chi \pm \mathrm{DP}$ & $20,2 \pm 4,4$ & $20,3 \pm 5,2$ & $22,7 \pm 4,5$ & $22,0 \pm 4,8$ & $0,0925^{* *}$ \\
\hline Mediana & 19,3 & 18,6 & 23,7 & 21,7 & \\
\hline$\%$ PA/PI & & & & & \\
\hline$\chi \pm \mathrm{DP}$ & $89,7 \pm 18,1$ & $90,2 \pm 18,4$ & $99,0 \pm 20,2$ & $85,6 \pm 24,5$ & $0,1494^{* *}$ \\
\hline Mediana & 89,5 & 86,1 & 101,3 & 88,2 & \\
\hline CB (cm) & & & & & \\
\hline$\chi \pm \mathrm{DP}$ & $24,8 \pm 3,8$ & $24,5 \pm 4,7$ & $27,1 \pm 4,0$ & $26,3 \pm 4,3$ & $0,0471^{* *}$ \\
\hline Mediana & 25,0 & 24,0 & 27,0 & 26 & \\
\hline PCT (mm) & & & & & \\
\hline$\chi \pm \mathrm{DP}$ & $13,6 \pm 6,9$ & $11,0 \pm 5,9$ & $15,7 \pm 7,2$ & $14,3 \pm 7,1$ & $0,1697 * *$ \\
\hline Mediana & 11,0 & 10,0 & 15,5 & 12,0 & \\
\hline CMB $\left(\mathrm{mm}^{2}\right)$ & & & & & \\
\hline$\chi \pm \mathrm{DP}$ & $205,8 \pm 34,8$ & $219,6 \pm 37,4$ & $222,0 \pm 38,9$ & $218,9 \pm 38,1$ & $0,1545^{* *}$ \\
\hline Mediana & 201,6 & 203,8 & 210,8 & 206,6 & \\
\hline AMB $\left(\mathbf{m m}^{2}\right)$ & & & & & \\
\hline$\chi \pm \mathrm{DP}$ & $3461,4 \pm 1289,9$ & $3644,2 \pm 1395,5$ & $4040 \pm 1432$ & $3927 \pm 1407$ & $0,1545^{* *}$ \\
\hline Mediana & 3237,3 & 3309,4 & 3554 & 3400 & \\
\hline $\mathrm{AAB}\left(\mathrm{mm}^{2}\right)$ & & & & & \\
\hline$\chi \pm \mathrm{DP}$ & $1405 \pm 987,6$ & $756 \pm 1091$ & $1923 \pm 945,2$ & $1746 \pm 946,9$ & $0,0955^{* *}$ \\
\hline Mediana & 1267 & 197,0 & 1827 & 1543,0 & \\
\hline
\end{tabular}

* Teste Exato de Fischer

** Teste de Kruskal-Wallis

$\mathrm{TI}=$ Tempo de internação; $\mathrm{IMC}=$ Índice de Massa Corporal; \% PA/PI = Porcentagem de adequação do Peso Atual em relação ao Peso Ideal; $\mathrm{CB}=$ Circunferência Braquial; $\mathrm{PCT}=$ Prega Cutânea do Tríceps; $\mathrm{CMB}=$ Circunferência Muscular do Braço, $\mathrm{AMB}=$ Área Muscular do Braço, $\mathrm{AAB}=$ Área Adiposa do Braço. 
Não foi observada diferença estatisticamente significante entre os pacientes portadores dos três grupos de neoplasias, quanto aos resultados dos exames laboratoriais (Tabela 2).

TABELA 2 - Indicadores laboratoriais, de consumo e necessidade energética da população estudada.

\begin{tabular}{|c|c|c|c|c|c|}
\hline & \multicolumn{5}{|c|}{ Neoplasias } \\
\hline & Esôfago & Estômago & $\begin{array}{c}\text { Cólon e } \\
\text { Reto }\end{array}$ & Total & $P$ \\
\hline $\begin{array}{l}\text { Linfócitos } \\
\left(\text { céls/mm } / \mathrm{mm}^{3}\right) \\
\mathrm{X} \pm \mathrm{DP} \\
\text { Mediana }\end{array}$ & $\begin{array}{c}1259,4 \pm 46 \\
7,8 \\
1340\end{array}$ & $\begin{array}{c}1500,6 \pm 93 \\
7,7 \\
1340\end{array}$ & $\begin{array}{c}1487,5 \pm 59 \\
4,5 \\
1545,5\end{array}$ & $\begin{array}{c}1491,2 \pm 69 \\
0,2 \\
1522\end{array}$ & 0,4391 \\
\hline $\begin{array}{l}\begin{array}{l}\text { Eritrócitos } \\
\text { (milhões } / \mathrm{mm}^{3} \text { ) }\end{array} \\
\mathrm{X} \pm \mathrm{DP} \\
\text { Mediana }\end{array}$ & $\begin{array}{c}3,8 \pm 1,1 \\
3,4\end{array}$ & $\begin{array}{c}4,4 \pm 1,1 \\
4,6\end{array}$ & $\begin{array}{c}4,3 \pm 0,8 \\
4,3\end{array}$ & $\begin{array}{c}4,2 \pm 0,9 \\
4,4\end{array}$ & 0,7730 \\
\hline $\begin{array}{l}\text { Hematócrito } \\
(\%) \\
\mathrm{X} \pm \mathrm{DP} \\
\text { Mediana }\end{array}$ & $\begin{array}{c}35,9 \pm 9,3 \\
4,1\end{array}$ & $\begin{array}{c}30,7 \pm 8,9 \\
29,8\end{array}$ & $\begin{array}{c}36,1 \pm 7,42 \\
36\end{array}$ & $\begin{array}{c}34,5 \pm 8,1 \\
33\end{array}$ & 0,3177 \\
\hline $\begin{array}{l}\text { Leucócitos } \\
\left(\text { céls } / \mathrm{mm}^{3}\right) \\
\mathrm{X} \pm \mathrm{DP} \\
\text { Mediana }\end{array}$ & $\begin{array}{c}9,3 \pm 3,2 \\
10,3\end{array}$ & $\begin{array}{c}10,5 \pm 2,7 \\
11,2\end{array}$ & $\begin{array}{c}9,26 \pm 2,6 \\
9,1\end{array}$ & $\begin{array}{c}9,5 \pm 2,6 \\
9,8\end{array}$ & 0,5968 \\
\hline $\begin{array}{l}\text { Hemoglobina } \\
(\mathrm{g} / \mathrm{dl}) \\
\mathrm{X} \pm \mathrm{DP} \\
\text { Mediana }\end{array}$ & $\begin{array}{c}11,8 \pm 3,2 \\
10,3\end{array}$ & $\begin{array}{c}9,6 \pm 3,1 \\
8,8\end{array}$ & $\begin{array}{c}11,7 \pm 2,6 \\
12,1\end{array}$ & $\begin{array}{c}11,1 \pm 2,8 \\
9,8\end{array}$ & 0,2115 \\
\hline CEH (Kcal) & & & & & \\
\hline $\begin{array}{l}\chi \pm \text { DP } \\
\text { Mediana }\end{array}$ & $\begin{array}{c}1566,3 \pm 352,2 \\
1709,2\end{array}$ & $\begin{array}{c}1288,8 \pm 740,8 \\
1319,6\end{array}$ & $\begin{array}{c}1557,3 \pm 623,1 \\
1588,8\end{array}$ & $\begin{array}{c}1480,6 \pm 656,3 \\
1458,3\end{array}$ & 0,4259 \\
\hline GET (Kcal) & & & & & \\
\hline$\chi \pm \mathrm{DP}$ & $2226,3 \pm 444,8$ & $2000,6 \pm 241,5$ & $1953,5 \pm 473,2$ & $1966,9 \pm 416,2$ & 0,0774 \\
\hline $\begin{array}{l}\text { Mediana } \\
\% \mathrm{CEH} / \mathrm{GET}\end{array}$ & 2173,7 & 2001,08 & 2055,4 & 2055,4 & \\
\hline$\chi \pm \mathrm{DP}$ & $72,8 \pm 23,9$ & $64,9 \pm 34,7$ & $80,8 \pm 28,3$ & $76,3 \pm 30,9$ & 0,4311 \\
\hline Mediana & 70,6 & 66,2 & 80,7 & 77,9 & \\
\hline
\end{tabular}

$\mathrm{CEH}=$ Consumo energético habitual, GET $=$ Gasto energético total.

$\% \mathrm{CEH} / \mathrm{GET}=$ Porcentagem de adequação entre o consumo energético habitual e o gasto energético total.

*Teste de Kruskal-Wallis

Observou-se (Tabela 2) que os portadores de neoplasia de estômago apresentaram CEH (kcal) inferior aos demais, porém, sem diferença significativa entre eles $(P=0,4259)$. Consequentemente, este grupo apresentou a menor porcentagem de adequação do $\mathrm{CEH}$ em relação a GET, embora sem diferença significativa $(P=0,4311)$.

Apenas $10,7 \%$ da amostra estudada apresentou CEH superior a $2000 \mathrm{kcal}$, e $28,6 \%$ inferior a $1000 \mathrm{kcal}$, evidenciando aqui a redução do $\mathrm{CEH}$ que acomete normalmente estes pacientes (Tabela 3 ). A dieta mais prescrita nesta clientela foi a enteral, em $35,7 \%$ deles, e chamou atenção a perda ponderal ocorrida durante a internação em $46,4 \%$ dos pacientes estudados; esta situação foi mais prevalente nos portadores de neoplasia de cólon e reto. A maioria dos pacientes recebeu atendimento nutricional no início e durante a internação e na alta hospitalar. Enfim, só foi constatada diferença estatística entre os três grupos de neoplasia para a $\mathrm{CB}$, apontando que pacientes portadores de neoplasia de estômago, seguidos dos portadores de neoplasia de esôfago, apresentam CB mais reduzida.
TABELA 3 - Consumo energético, dieta prescrita, alteração ponderal e intervenção nutricional, durante a internação

\begin{tabular}{lcccc}
\hline & \multicolumn{4}{c}{ Neoplasias } \\
& $\begin{array}{c}\text { Esôfago } \\
\text { N(\%) }\end{array}$ & $\begin{array}{c}\text { Estômago } \\
\mathbf{N}(\%)\end{array}$ & $\begin{array}{c}\text { Cólon e Reto } \\
\mathbf{N}(\%)\end{array}$ & $\begin{array}{c}\text { Total } \\
\mathbf{N}(\%)\end{array}$ \\
\hline Consumo energético habitual & & & & \\
$<1000$ Kcal & $1(12,5)$ & $3(37,5)$ & $4(33,3)$ & $8(28,6)$ \\
$\geq 1000$ Kcal e $\leq 1500 \mathrm{Kcal}$ & $2(25,0)$ & $3(37,5)$ & $3(25,0)$ & $8(28,6)$ \\
$>1500$ Kcal e $\leq 2000 \mathrm{Kcal}$ & $5(62,5)$ & $1(12,5)$ & $3(25,0)$ & $9(32,2)$ \\
$>$ 2000 Kcal & - & $1(12,5)$ & $2(16,7)$ & $3(10,7)$ \\
Dieta prescrita & & & & \\
Geral & - & - & $2(16,7)$ & $2(7,1)$ \\
Branda & - & - & $1(8,3)$ & $1(3,6)$ \\
Leve & - & $1(12,5)$ & $3(25,0)$ & $4(14,3)$ \\
Líquida & $7(87,5)$ & $3(37,5)$ & $3(25,0)$ & $4(14,3)$ \\
Enteral & $1(12,5)$ & $3(37,5)$ & $3(25,0)$ & $7(25,0)$ \\
Jejum & & & & \\
Alteração ponderal & $3(37,5)$ & $4(50,0)$ & $4(33,3)$ & $11(39,3)$ \\
Manutenção ponderal & $2(25,0)$ & $1(12,5)$ & $1(8,3)$ & $4(14,3)$ \\
Ganho ponderal & $3(37,5)$ & $3(37,5)$ & $7(58,3)$ & $13(46,4)$ \\
Perda ponderal & & & & \\
Intervenção nutricional & - & $2(25,0)$ & $1(8,3)$ & $3(10,7)$ \\
$1 *$ & $3(37,5)$ & $2(25,0)$ & $6(50,0)$ & $11(39,3)$ \\
1 e 2** & $5(62,5)$ & $4(50,0)$ & $5(41,7)$ & $14(50,0)$ \\
1,2 e 3*** & & &
\end{tabular}

* Na internação, ** Na internação e durante a internação, *** Na internação, durante a internação e na alta hospitalar.

\section{DISCUSSÃO}

De acordo com o tipo e o estágio das neoplasias do trato-digestório, podem ocorrer algumas alterações orgânicas que contribuem para a redução do bem estar geral e da qualidade de vida do paciente ${ }^{27}$, sendo a desnutrição complicação frequente em pacientes com neoplasias (40$80 \%$ dos casos), o que está associado de forma significativa à morbidade e mortalidade ${ }^{3}$. Em estudo com 30 pacientes (44\% com neoplasia de cólon e 17\% com neoplasia gástrica e esofágica), observou-se em avaliação posterior, $37 \%$ dos pacientes em bom estado nutricional, $60 \%$ em desnutrição leve e $3 \%$ em desnutrição grave ${ }^{7}$. No presente estudo, foi encontrado IMC na amostra total de $22,0 \pm 4,8$, no entanto, quando segmentado por faixas dos pontos de corte, foi verificado que $50 \%$ apresentaram eutrofia e $25 \%$ baixo peso (mais prevalente nos portadores de neoplasia de estômago). Resultados semelhantes foram encontrados por Candela et al. ${ }^{3}$, com 53\% dos pacientes apresentando IMC entre 19-25, $7 \%$ com IMC inferior a 19 e $40 \%$ com IMC acima de 25 .

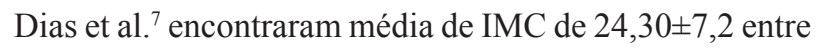
seus pacientes, em uma primeira análise e $23,15 \pm 6,4$, em segundo momento. Os dados aqui apresentados mostraram que $46,4 \%$ dos pacientes apresentaram perda de peso durante a internação. Estes índices vão de encontro aos resultados obtidos por Dias et al. ${ }^{7}$, que documentaram presença de perda ponderal, durante o período de um mês, em $55 \%$ dos pacientes, ganho ponderal em $35 \%$ deles, e manutenção ponderal em 10\% dos pacientes. Ravasco et al. ${ }^{27}$, avaliaram doentes neoplásicos em estágios avançados e mostraram que os portadores de tumores esofágicos e gástricos apresentaram maiores perdas de peso em relação 
aos que apresentavam tumores de cólon e reto. De forma geral, $85 \%$ dos pacientes com os estágios III e IV da doença apresentaram perdas superiores a $10 \%$ de seu peso habitual. Outro estudo, realizado por Cid Conde et al. ${ }^{5}$, analisou a prevalência e o grau de subnutrição entre pacientes com tumores digestivos no período pré-operatório e constatou que $53 \%$ dos pacientes avaliados tinham perdido $5 \%$ de seu peso habitual nos últimos três meses. A avaliação subjetiva global apontou prevalência de subnutrição em $50 \%$ dos pacientes $(29 \%$ com subnutrição moderada ou risco de subnutrição e $21 \%$ com subnutrição grave).

A perda de peso está, em grande parte, associada ao diagnóstico de neoplasia do trato digestório. A desnutrição e a perda ponderal é muito comum em pacientes que apresentam comprometimento do trato digestório, principalmente devido à baixa ingestão alimentar decorrente da própria sintomatologia da doença e de seu tratamento20. Em outro estudo realizado por Garcia et al. ${ }^{14}$, foi observado que a maior prevalência de desnutrição ocorreu em pacientes com neoplasias, e confirmou que as doenças neoplásicas (principalmente as do trato digestório), estão mais associadas com a desnutrição do que as não neoplásicas, sendo os idosos acima de 75 anos propensos a risco maior. A avaliação nutricional mostrou que $10 \%$ dos pacientes apresentaram-se sem depleção, $65 \%$ depleção leve e $25 \%$ moderada do estado nutricional. A perda ponderal e as alterações relacionadas à alimentação são comumente encontradas em pacientes com câncer avançado ${ }^{6}$.

Em relação a AMB, 46,4\% dos pacientes apresentaramse abaixo de P5, entre estes, a maioria dos portadores de neoplasia de esôfago, já quanto a PCT, a maioria apresentou eutrofia, e a CMB encontrada foi de 218,9 $\pm 38,1$, o que apontou valores abaixo de $\mathrm{P} 5$ para o sexo masculino e entre P25-50 para o sexo feminino. O estudo de Candela et al. ${ }^{3}$ revelou que $59 \%$ dos homens e $12 \%$ das mulheres também apresentaram valores da AMB inferiores aos recomendados. A média da PCT encontrada para homens foi de $11 \pm 4 \mathrm{~mm}$ e para as mulheres $19 \pm 6 \mathrm{~mm}$ e a média da CMB foi de $240 \pm 2 \mathrm{~mm}$ para homens e $220 \pm 3 \mathrm{~mm}$ para mulheres, caracterizando eutrofia, contrariando aqui os resultados obtidos no presente trabalho.

$\mathrm{Na}$ análise dos indicadores laboratoriais de hemoglobina, hematócrito e leucócitos, os valores encontrados foram semelhantes aos achados de Dias et al. ${ }^{7}$ estando os resultados de hemoglobina e hematócrito abaixo dos valores de referência nos dois estudos, isto provavelmente devido à baixa ingestão alimentar, má-absorção de nutrientes, além de sangramentos recorrentes devido às ulcerações comuns nesta situação. Já a contagem de leucócitos, que esteve dentro da normalidade no presente estudo, frequentemente está alterada em clientela que é submetida rotineiramente a sessões de radioterapia e quimioterapia ${ }^{23}$.

Estes dados apontaram que 28,6\% dos pacientes apresentaram CEH inferior a $1000 \mathrm{kcal}$, prevalecendo nesta faixa de consumo energético os portadores de neoplasia de estômago e de cólon e reto. É importante salientar aqui que os portadores de neoplasia de esôfago provavelmente apresentaram maior consumo energético por estarem sendo alimentados por alimentação enteral. Foi verificado prescrição de dieta enteral de forma mais acentuada na neoplasia de esôfago. Nestes casos, sintomas como odinofagia e disfagia, causados pelo tumor, são muito mais frequentes, e podem muitas vezes impedir a alimentação por via oral. No estudo de Candela et al. ${ }^{3}$, o CEH médio dos pacientes foi de $1740 \pm 500 \mathrm{kcal} / \mathrm{dia}$ e a NET média foi de $1800 \pm 200 \mathrm{kcal} / \mathrm{dia}$, de forma que $30 \%$ desses indivíduos apresentaram CEH inferior às suas necessidades energéticas diárias. Este fato deve-se provavelmente às condições que a própria doença determina ao paciente, tanto em termos nutricionais quanto metabólicos e funcionais, além de limitações físicas no curso da doença. Ravasco et al. ${ }^{27}$, também demonstraram que os sintomas presentes nas neoplasias do trato-digestório, são importantes determinantes da qualidade de vida dos pacientes, verificando assim que os portadores de neoplasias de esôfago apresentavam mais fadiga que os demais pacientes, enquanto que nos casos de estômago e cólon e reto, as náuseas e vômitos foram mais intensos. Para todos os diagnósticos, a dispnéia, a insônia e a anorexia foram mais prevalentes nos estágios mais avançados da doença e a diarréia foi mais prevalente nos indivíduos com tumores de cólon e reto. De acordo com Dicken et $\mathrm{al}^{8}$, $95 \%$ dos pacientes com câncer gástrico, apresentam anorexia e perda de peso. Ainda, a síndrome da anorexia-caquexia, pode ser observada em $80 \%$ dos pacientes oncológicos em estágios mais avançados, sendo este quadro uma das mais freqüentes causas de mortalidade entre estes indivíduos ${ }^{24}$.

No estudo realizado por Dias et al. ${ }^{7}$, foi observado redução da ingestão alimentar em $50 \%$ dos pacientes estudados, independente do fato deles apresentarem ou não sintomas gastrintestinais. A alteração na consistência da dieta foi observada em $25 \%$ dos indivíduos. De acordo com os dados de Prieto et al. $.^{25}, 50 \%$ dos pacientes com doenças do trato digestório permaneceram em jejum, sendo esta prescrição a mais prevalente entre os pacientes com neoplasia $(25,8 \%)$. Destes, $22,6 \%$ receberam dieta geral, $6,4 \%$ dieta pastosa, $16,1 \%$ dieta leve, e $9,7 \%$ dieta líquida, entre outros tipos de prescrições.

Em outro estudo ${ }^{21}$, verificou-se que a nutrição enteral foi utilizada somente em pacientes com neoplasias $(6,4 \%)$. Quanto à conduta dietoterápica adotada, a dieta leve foi prescrita em $6,4 \%$ dos pacientes, a dieta branda em $4,8 \%$, e a geral em $14,5 \%$, entre outros tipos específicos. $\mathrm{Na}$ avaliação de Garcia et al. ${ }^{14}$, a prescrição da dieta enteral também predominou naqueles indivíduos com diagnóstico de neoplasia $(44,4 \%)$, sendo que $42,1 \%$ das prescrições referiam-se à dieta geral, $10,5 \%$ à dieta branda, $21,5 \%$ à dieta leve, e 7,8\% à dieta líquida. A dieta enteral em pacientes portadores de neoplasia é muito comum, principalmente em pós-operatórios. Em metanálise recente1, referente a estudos realizados em pacientes oncológicos submetidos à operações gastrointestinais, foi demonstrado que os alimentados com dieta enteral apresentaram menores complicações pós-operatórias, como infecções e redução do tempo de internação hospitalar. Em pacientes com neoplasias do trato-digestório, a desnutrição grave está associada 
com o aumento da morbidade e mortalidade, redução da eficácia do tratamento e aumento do tempo de internação ${ }^{29}$. A intervenção nutricional deve ser implementada assim que o câncer for diagnosticado, e deve ser adaptada às necessidades individuais, considerando-se o tipo de tratamento oncológico, as condições clínicas e o estado nutricional ${ }^{4}$.

Os dados encontrados no presente trabalho permitiram concluir que todos os pacientes avaliados sofreram comprometimento em seu estado nutricional, sendo importante o acompanhamento nutricional durante o tratamento. Os pacientes portadores de neoplasias do estômago foram prejudicados em relação aos indicadores antropométricos e dietéticos, uma vez que de forma geral, apresentaram depleção de tecido adiposo (valores reduzidos de $\mathrm{AAB}$ ), além de menor \% PA/PI, e baixa adequação do $\mathrm{CEH}$ em relação a GET. Este tipo de paciente, normalmente, já tem o seu consumo alimentar bastante afetado, já que a intensidade e frequência dos sintomas (náuseas, vômitos, anorexia) decorrentes da doença são maiores, se comparados com os outros tipos de neoplasias. Os pacientes portadores de neoplasia esofágica apresentam limitação mecânica para ingestão alimentar, o que justifica o maior comprometimento em termos de perda de massa magra. Assim, estes pacientes demonstraram alterações nos indicadores antropométricos (CMB, AMB e AAB) e laboratoriais (eritrócitos, hemoglobina e hematócrito). Os portadores de neoplasia de cólon e reto apresentaram maiores perdas ponderais durante a internação, possivelmente devido ao $\mathrm{CEH}$ reduzido e maior prevalência de prescrições de dietas líquidas, leve e jejum durante a internação, fatores estes que podem estar associados a frequência maior de diarréias e má-absorção, muito comum nesta clientela. Entretanto, estes indivíduos obtiveram os melhores valores antropométricos, o que não significa que não estejam em risco para a desnutrição, uma vez que a perda ponderal, a longo prazo, poderia afetar diretamente estes indicadores.

\section{CONCLUSÃO}

Pacientes com diagnóstico de neoplasia de trato digestório apresentam comprometimento no estado nutricional e necessitam de acompanhamento nutricional no decorrer do tratamento destas enfermidades.

Leandro-Merhi VA, Tristão AP, Moretto MC, Fugulin NM, Portero-McLellan KC, Aquino JLB. Comparative study of nutritional status indicators among patients with gastrointestinal neoplasms. ABCD Arq Bras Cir Dig 2008;21(3):114-9

ABSTRACT - Background - Cancer nowadays is considered one of the biggest health problem all over the world and the digestive and respiratory tumors are the most frequent among them. Aim - To compare nutritional indicators among patients with esophageal, stomach and intestinal neoplasms. Methods - A comparative study was carried out assessing indicators of nutritional status (anthropometry, biochemistry and diet) of 28 patients with digestive neoplasms. Results - Groups were statistically paired by gender, biochemical tests, food intake and anthropometrics measurements, except for the upper arm circumference $(P=0.0471)$. Weight loss was verified for $46.4 \%$ of the studied patients and the tube diet was the most prevalent dietary prescription for the patients, especially among those with esophageal neoplasms. Conclusion - Patients with esophageal, stomach and intestinal neoplasms have poor nutritional status and need continuous dietary care during the treatment of their diseases.

HEADINGS - Esophageal neoplasms. Stomach neoplasms. Colonic neoplasms. Nutritional status.

\section{REFERÊNCIAS}

1. Alvarez CS, Ruiz RN, Garcia VM. Soporte nutricional en el paciente con neoplasia digestiva. Nutrición Hospitalaria 2005; 20(2):38-40.

2. Burr ML, Phillips MK. Anthropometric norms in the elderly. Br J Nutr 1984; 51:165-9.

3. Candela CG, Luengo LM, Roque VM, Iglesias C, Zamora P, Barón RG. Valoración global subjetiva en el paciente neoplásico. Nutrición Hospitalaria 2003; 6(18): 353-357.

4. Caro MM, Laviano A, Pichard C, Candela CG. Relationship between nutritional intervention and quality of life in cancer patients. Nutr Hosp 2007; 22(3):33750 .

5. Cid Conde L, Fernández López T, Neira Blanco P, Arias Delgado J, Varela Correa JJ, Gómez Lorenzo FF. Hyponutrition prevalence among patients with digestive neoplasm before surgery . Nutr Hosp. 2008; 23(1):46-53.

6. Dewey A, Dean T. Nurses' management of patients with advanced cancer and weight loss: part 2. Int J Palliat Nurs. 2008; 14(3):132-8.

7. Dias VM, Barreto APM, Coelho SC, Ferreira FMB, Vieira GBS, Cláudio MM, Silva PD G. O grau de interferência dos sintomas gastrintestinais no estado nutricional do paciente com câncer em tratamento quimioterápico. Rev Bras Nut Clín 2006; 3(21): 211-218.

8. Dicken BJ, Bigam DL, Cass C, Mackey JR, Joy AA, Hamilton SM. Gastric adenocarcinoma: review and considerations for future directions. Annals of Surgery $2005 ; 241(1): 27-39$

9. Esper D, Harb W. The cancer cachexia syndrome: a review of metabolic and clinical manifestations. Nutr Clin Pract 2005; 20: 369-76.

10. Fernandes CL, Pucca L, Matos D. Diagnóstico e Tratamento de Tumores Carcinóides do Trato Digestivo. Rev Assoc Med Bras 2002; 48(1): 87-92.

11. Fleury. Manual 15. F de Exames 2008/2009. Laboratório Fleury. São Paulo, 2008
12. Frisancho AR. Anthropometric standards for the assessment of growth and nutritional status. Michigan: The University of Michigan Press; 1990.

13. Frisancho AR. New norms of upper limb fat muscle areas for assessment of nutritional status. Am J Clin Nutr 1981; 34:2540-5.

14. Garcia RWD, Leandro-Merhi VA, Pereira AM. Estado nutricional e sua evolução em pacientes internados em clínica médica. Rev Bras Nut Clín 2004; 19(2): 59-63.

15. Garófolo A, Avesani CM, Camargo KG, Barros ME, Silva SRJ, Taddei JAAC, Sigulem D M. Diet and cancer: an epidemiological view. Rev Nutrição 2004; 17(4):491-505.

16. Gray GE, Gray LK. Anthropometric measurements and their interpretation: principles, practices and problems. J Am Diet Assoc 1980; 77:534-9.

17. Guerra MR, Moura Gallo CV, Mendonça GAS. Risco de câncer no Brasil: tendências e estudos epidemiológicos mais recentes. Rev Bras Cancerologia 2005; 51(3):227-234.

18. Heymsfield SB, Thighe A, Wang ZM. Nutritional assessment by anthropometric and biochemical methods. In Shills ME, Olson JA, Shije M. Modern Nutrition in health and disease. 9th ed. Baltimore: Williams \& Wilkins; 1999. p. 812-41.

19. INCA-Instituto Nacional do Câncer: Estimativa 2008-Incidência de câncer no Brasil. Disponível em: http://www.inca.gov.br/. Acesso em: 23/01/ 2009.

20. Leandro-Merhi VA, Garcia RWD, Mônaco DV, Oliveira MRM. Comparación del estado nutricional, consumo alimentício y tiempo de hospitalización de pacientes de dos hospitales, uno público y otro privado. Nutr Hosp. 2006; 21(1):32-7.

21. Leandro-Merhi VA, Mônaco DV, Lazarini ALG, Yamashiro A, Maciel AC; Estado nutricional de pacientes hospitalizados em um hospital privado. Rev Bras Nut Clín 2004; 19(3): 116-122. 
22. Lipschitz DA. Screening for nutritional status in the elderly. Prim Care 1994; 22(1):55-67.

23. Martins C, Moreira SM, Pierosan SR. Interações Droga Nutriente. $2^{\mathrm{a}}$ ed. Curitiba: Nutroclínica, 2003.

24. Perboni S, Inui A. Anorexia in cancer: role of feeding-regulatory peptides. Philosophical Transactions of de Royal Society Biological Sciences 2006; 361(1471): 1281-1289.

25. Prieto DB, Leandro-Merhi VA, Mônaco DV, Lazarini ALG. Intervenção nutricional de rotina em pacientes de um hospital privado. Rev Bras Nutr Clin. 2006; 21(3):181-7.

26. Ravasco P, Grillo IM, Camilo M. Cancer wasting and quality of life react to early individualizad nutricional counselling. Clin Nutrition 2007; 26: 7-15.

27. Ravasco P, Monteiro-Grillo I, Vidal PM, Camilo ME. Qualidade de vida em pacientes com cancro gastrintestinal. Qual é o impacto da nutrição? Acta Medica Portuguesa 2006; 19(2):189-196.
28. SAS System for Windows (Statistical Analysis System), versão 9.1.3 Service Pack 3. SAS Institute Inc, 2002-2003, Cary, NC, USA.

29. Senesse P, Assenat E, Schneider S, Chargari C, Magné N, Azria D, Hébuterne X. Nutritional support during oncologic treatment of patients with gastrointestinal câncer: who could benefit? Cancer Treat Rev 2008; 34(6):568-75.

30. Universidade Federal de São Paulo. Escola Paulista de Medicina. Programa de Apoio a Nutrição (NUTWIN)-programa de computador, versão 1.5. São Paulo: UNIFESP; 2002.

31. World Health Organization. The World Health Report 1998: Life in the 21st century a vision for all. Geneva: WHO; 1998. p. 61-111.

32. Yaghoobi M, Rakhshani N, Sadr F, Bijarchi R, Joshaghani Y, Mohammadkhani A, Attari A, Akbari R, Hormazdi M, Malekzadeh R.. Hereditary risk factors for the development of gastric cancer in younger patients. BMC Gastroenterology 2004; 4(28):141-145.

Fonte de financiamento: não há Conflito de interesse: não há Recebido para publicação: 15/01/2008 Aceito para publicação: 21/03/2008 